\title{
PREVALÊNCIA DA EPILEPSIA ENTRE OS ÍNDIOS BAKAIRI DO ESTADO DO MATO GROSSO, BRASIL
}

\author{
Moacir Alves Borges' ${ }^{1}$, Edir Pina de Barros², \\ Dirce Maria Trevisan Zanetta ${ }^{3}$, Ana Paula Pina Borges ${ }^{4}$
}

\begin{abstract}
RESUMO - O objetivo deste estudo, tipo corte transversal, com base populacional foi determinar a prevalência da epilepsia, mediante delineamento em duas etapas, entre os índios Bakairi que residem às margens do rio Paranatinga, no planalto central no Estado do Mato Grosso/Brasil. O estudo foi feito de porta-a-porta em 103 casas, abrangendo 483 dos 546 indivíduos que aí vivem. A sensibilidade do instrumento foi de $88,8 \%$ (IC95\%, 84,9-93) a especificidade de 97,8\% (IC-95\%, 95-98), a prevalência de epilepsia ativa foi de 12,4\%o e a inativa, de $6,2 \%$. A prevalência de epilepsia foi considerada alta, provavelmente em consequência do fator de risco familial $(p=0,04)$, uma vez que outros não foram relevantes.
\end{abstract}

PALAVRAS-CHAVE: epilepsia, prevalência, fator de risco, índios Bakairi.

\section{Prevalence of epilepsy in Bakairi indians from Mato Grosso State, Brazil}

ABSTRACT - The purpose of this cross-sectional study was to evaluate the prevalence of epilepsy in a population based sample: the Bakairi indians, who live on the banks of Paranatinga river in the central plateau of Mato Grosso state, Brazil. This population is composed of 483 inhabitants. The study was outlined in two stages, and the investigation was door-to-door in a total of 103 houses. The results of the instrument showed a sensitivity of $88 \%$ (Cl- 95\%, 84.9-93), specificity of $97.8 \%$ (Cl-95, 95-98). The prevalence of active epilepsy was $12.4 \%$, and inactive was $6.2 \%$. This prevalence of the epilepsy was considered high probably due to some familial risk factor $(p=0.04)$ among those Indians, while other factors were not important.

KEY WORDS: epilepsy, prevalence, risk factor, Bakairi indian.

A epilepsia é o terceiro dos distúrbios neurológicos mais comuns. O referido transtorno ocasiona problema de saúde pública na comunidade e como tal deve ser abordado ${ }^{1-3}$. A investigação epidemiológica para determinar a freqüência, a distribuição e os fatores de risco desta entidade se faz necessária e deve ser representativa entre as diversas populações de um país ${ }^{4}$. Os resultados dos estudos epidemiológicos sobre epilepsia têm apontado ampla faixa de variação como valores entre $0,9 \%$ o habitantes ${ }^{5}$ e $57 \% 0^{6}$ para a prevalência e $26 / 100000 /$ ano $^{7,8}$ para a incidência. As referidas variações podem representar diferenças entre as populações estudadas em consequência de fatores tais como o serviço inadequado de saúde pública (particularmente os cuidados perinatais), o programa de vacinação, a exposição a agentes infecciosos ou infestantes como cisticercose e o trauma crânio-encefálico, embora questões metodológicas possam contribuir ou ser a razão de resultados tão distintos ${ }^{4}$. O fato é que numerosos estudos têm mostrado que os países em desenvolvimento apresentam prevalência de epilepsia significantemente maior que os países desenvolvidos. Gracia et al. ${ }^{6}$, no final da década de oitenta, na República do Panamá, onde a prevalência populacional de epilepsia era de $22 \%$, analisaram uma comunidade indígena já em contato com a população branca de longa data, porém sem miscegenação. Encontraram alta prevalência de epilepsia (57\%o), sendo o fator de risco mais importante a história com antecedentes familiares de epilepsia $(R R=14)$.

O Brasil, embora, com poucos estudos epidemiológicos, apresenta prevalência de epilepsia similar aos países em desenvolvimento. Mesmo nas áreas cosmopolitas onde há acesso à medicina com tecnologia de ponta, a prevalência é alta por não atingir o

Departamento de Ciências Neurológicas da Faculdade de Medicina Rio Preto (FAMERP), São José do Rio Preto SP, Brasil: ${ }^{1}$ Professor Assistente Mestre, responsável do Serviço de Neurofisiologia Clínica da FAMERP; ${ }^{2}$ Antropóloga Professora Doutora Adjunta da Faculdade Federal do Mato Grosso (UFMT), Cuibá MT, Brasil; ${ }^{3}$ Professora Doutora Chefe do Departamento de Epidemiologia e Saúde Coletiva da FAMERP; ${ }^{4}$ Acadêmica da FAMERP.

Recebido 11 Junho 2001, recebido na forma final 3 Setembro 2001. Aceito 21 Setembro 2001. 
sistema de saúde pública a totalidade da população ${ }^{2,3}$, além dos contigentes migratórios das zonas rurais ou cidades pequenas que não dispunham de saneamento básico satisfatório. A respeito das comunidades indígenas brasileiras, atualmente não há estudos referentes aos aspectos epidemiológicos sobre epilepsia.

O objetivo do presente estudo foi determinar a prevalência e os principais fatores de risco da epilepsia entre os índios Bakairi.

\section{MÉTODO}

\section{Estudo populacional: Nação Bakairi}

Os Bakairi, povo de língua Karíb, vivem em terras Indígenas denominadas Bakairi e Santana, situadas na região do cerrado norte mato-grossense, sudoeste do Alto Xingu. A Terra Indígena Bakairi localiza-se às margens do rio Paranatinga (municipío de Paranatinga) e Santana nas cabeceiras do rio Arinos (município de Nobres). Os rios Paranatinga ou Telles Pires e Arinos pertencem à Bacia Amazônica (Fig 1). Na Terra Indígena Bakairi (município de Paranatinga) somam-se 546 indivíduos distribuídos em sete aldeias. Em Santana (município de Nobres) vivem 166 Bakairi, em três aldeias. Além destes 712 Bakairi, existem alguns que residem em cidades, dentre elas Nobres, Paranatinga e Cuiabá.

Os Bakairi residem em casas de pau-a-pique barroteadas. Sobrevivem da agricultura (mandioca e arroz), da pesca, da criação de gado, assumindo a caça e a coleta, um papel secundário e complementar.
Concorrem, para a sua sobrevivência, os proventos oriundos das aposentadorias e salários por trabalhos prestados nas aldeias e às prefeituras dos municípios onde vivem (professores e merendeiras) e à Fundação Nacional do Índio (FUNAI), órgão governamental responsável pela tutela dos indígenas brasileiros, vinculado ao Ministério da Justiça. Cada aldeia tem o seu próprio líder e possui relativa autonomia econômica e política frente a outras unidades sociais da mesma ordem. Os interesses comuns são discutidos em reuniões de líderes ou caciques. Eles são alfabetizados na própria língua materna - que todos praticam - tendo como segunda língua o português, que falam fluentemente.

$\mathrm{O}$ atendimento à saúde, inicialmente sob a responsabilidade da Fundação Nacional do Índio, hoje está a cargo da Fundação Nacional de Saúde (FUNASA), órgão do Ministério da Saúde que em novembro de 1999, através do Convênio 349/99, firmou parceria com o Instituto de Apoio ao Desenvolvimento Humano e do Meio Ambiente/Tropicus, organização não governamental voltada a atenção primária à saúde dos povos indígenas de uma parcela do Distrito Sanitário Especial Indígena de Cuiabá (DSEICUIABA), dentre os Baikiri. Essa entidade se encarrega do atendimento médico-odontológico ambulatorial, além de realizar e manter $\mathrm{o}$ arquivo das patologias encontradas. Além disso, atuam em cada uma das aldeias, os Agentes Indígenas de Saúde (AIS), egressos dos quadros da FUNAI e hoje da FUNASA, que prestam os primeiros atendimentos primários de saúde - processo que envolve os xamãs ou curandeiros.

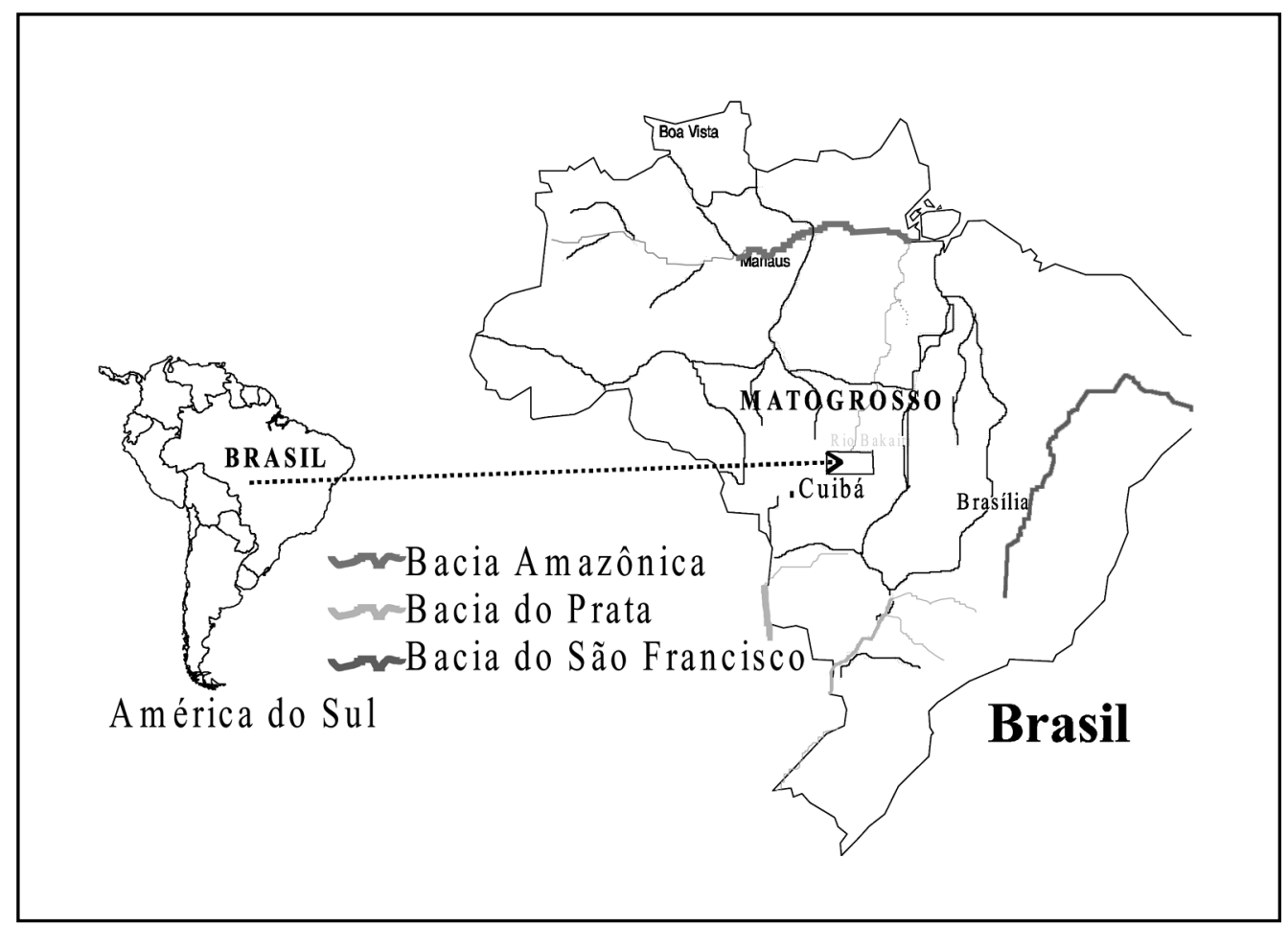

Fig 1. Mapa mostrando a localização das terras dos Bakairis. 


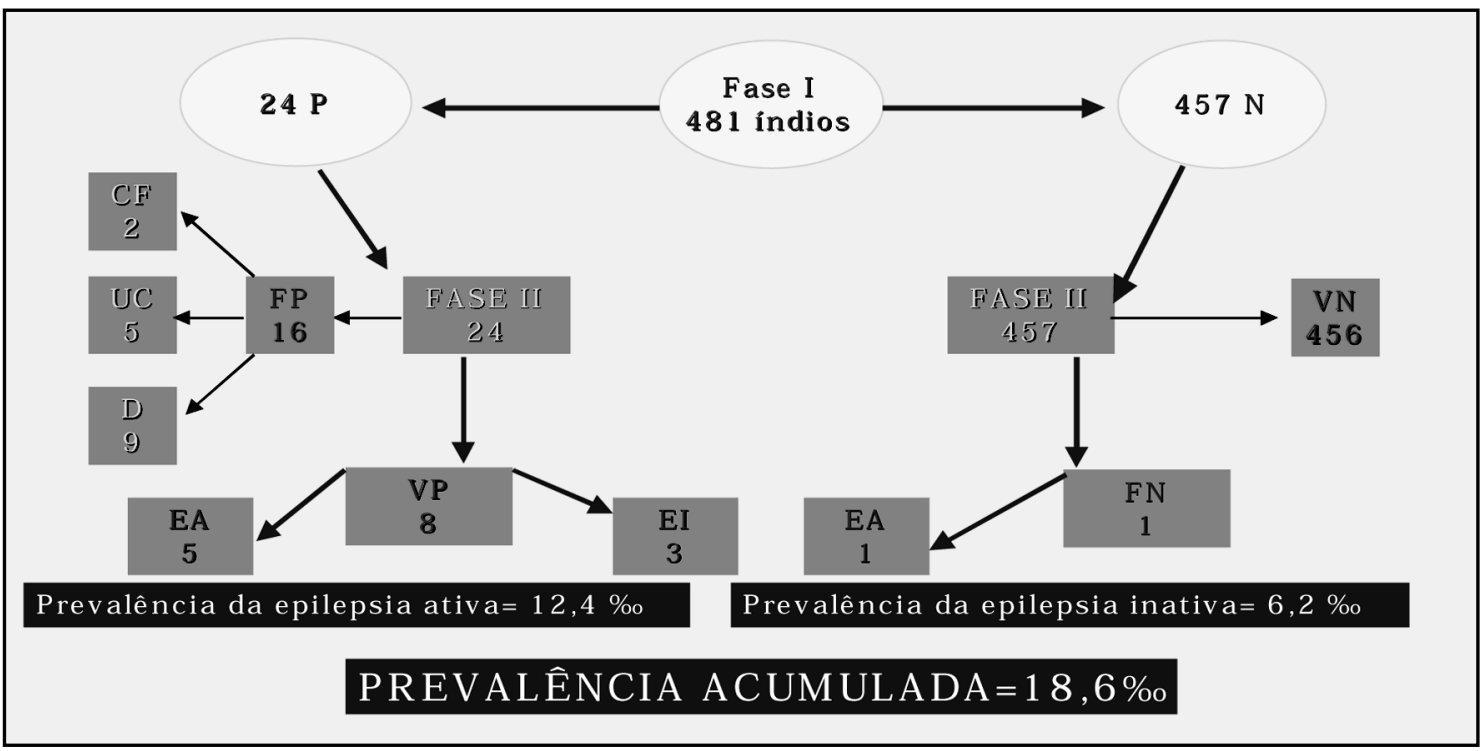

Fig 2. Diagrama das fases da pesquisa sobre prevalência da epilepsia entre os índios Bakairi. $P$, positivo; $C F$, crise febril; UC, crise única; VP, verdadeiro positivo; FP, falso positivo; VN, verdadeiro negativo; FN, falso negativo, $N$, negativo; EA, epilepsia ativa; El, epilepsia inativa.

\section{Pesquisa populacional}

A primeira fase do estudo observacional populacional tipo corte transversal foi executado mediante questionário de rastreamento de epilepsia de Placencia et al. ${ }^{9}$, (sensibilidade de $79.3 \%$ e especificidade de $96,3 \%$ ), modificado para 8 perguntas, já testado na cidade de São José do Rio Preto, cujo estudo piloto mostrou sensibilidade 95,8\% (IC-95\%;94,5-97) e especificidade de 97,8\% (IC-95\%;96,9$98,7)$. Foi aplicado na população da Terra Indígena Bakairi às margens do rio Paranatinga, constituída de 546 índios, tendo sido objeto deste estudo apenas 483 (88,46\%) que na atualidade residem em 103 casas, com início em julho e término em dezembro de 2000.

$O$ instrumento foi aplicado de porta-em-porta por uma acadêmica do $3^{\circ}$ ano da Faculdade de Medicina de São José do Rio Preto, acompanhada e orientada pelo AIS e supervisionada por um antropólogo ${ }^{10,11}$. O objetivo, dessa etapa, foi identificar todos os casos referentes às crises epilépticas, às crises únicas, às sintomáticas agudas e febris. Foram considerados dois tipos de situações: o primeiro, cujo questionário aplicado revelou pelo menos uma questão com resposta afirmativa, tendo sido considerado teste POSITIVO e o segundo em que todas as respostas foram negativas, considerou-se teste NEGATIVO.

A partir dos resultados destes questionários, foi aplicado o padrão ouro: história clínica, exame neurológico ${ }^{12}$, eletrencefalograma (EEG) ${ }^{13}$. Aplicou-se o padrão ouro por um neurofisiologista com experiência em epilepsia em casos positivos com a finalidade de se determinar os casos verdadeiros e os falsos positivos. Em seguida, o protocolo padrão foi aplicado em 50 indivíduos, escolhidos ao acaso, dos indivíduos considerados negativos para se determinar os verdadeiros e falsos negativos.
Todos os resultados foram cruzados com os registros médicos do Instituto Trópicus, com auxílio do AIS. Também foram coletados os dados sobre idade, sexo, tempo de crise, frequência, história familial de epilepsia, tipos e síndromes epilépticas, terapia e controle de crises.

\section{Classificação dos pacientes de acordo}

com o tipo e síndrome epiléptica

Foram adotados os critérios de classificação da "International League against Epilepsy" (ILAE) ${ }^{14,15}$, quanto às crises e síndromes epilépticas na elaboração do protocolo padrão. Além disso, incluíram-se apenas aquelas pessoas que tiveram, pelo menos, duas crises não provocadas e com intervalo superior a $24 \mathrm{~h}$ entre a primeira e a segunda crise. Foi adotado o critério de epilepsia ativa de Jacoby $^{16}$, isto é, pelo menos uma crise nos dois últimos anos. Utilizou-se o critério de Palmine e Da Costa ${ }^{17}$ para diferenciar a epilepsia de crises epilépticas, segundo o qual, a primeira constitui manifestações de diferentes distúrbios cerebrais (algumas vezes sua principal manifestação), e a segunda é apenas uma das manifestações da entidade.

A etiologia da epilepsia foi classificada de acordo com as normas da "Commission Epidemiology and Prognosis" da $\operatorname{ILAE}^{18}$, conforme segue: idiopática refere-se à epilepsia que se enquadra em umas das síndromes epilépticas (presumidamente genética) e ocorre na ausência de quaisquer anormalidades neurológicas; sintomática refere-se à epilepsia que ocorre na presença de uma agressão remota ou a uma prévia desordem que seja reconhecidamente associada a fator de risco da epilepsia e, finalmente, o termo criptogênico que é usado para a ausência de causa identificável, e o paciente não reunir critério para a síndrome epiléptica idiopática. A Figura 2 mostra o diagrama do delineamento do estudo. 


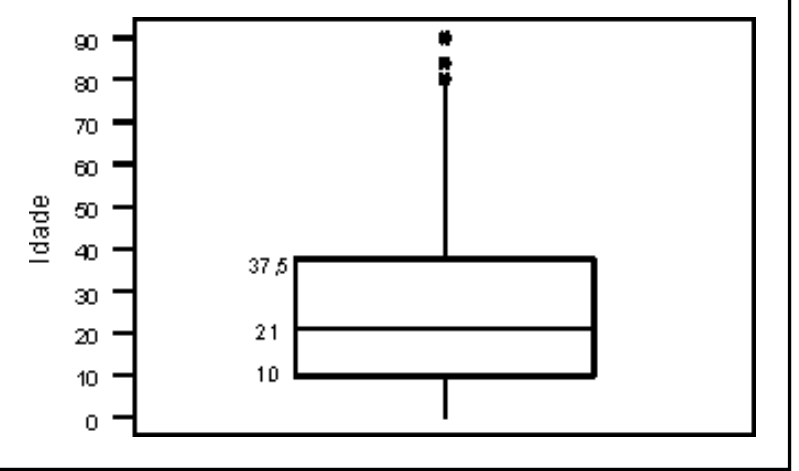

Fig 3. Distribuição das idades entre os índios Bakairi.

\section{Avaliação do controle das crises}

O controle das crises foi avaliado baseando-se nos critérios de Semah et al. ${ }^{19} \mathrm{em} 4$ grupos: fácil controle (pacientes sem crises usando monoterapia nas dosagens usuais diárias); crise de difícil controle (pacientes sem crises sob politerapia em altas doses diárias); sem controle de crises e controle não definido (por exemplo, por evolução inadequada ou falta de dados).

Foi fornecido a todos índios participantes uma fixa contendo o consentimento informado.
Os resultados foram armazenados em planilha do Excel para os cálculos estatísticos do $\chi^{2}$, e teste exato de Fisher para análise estatística de amostras pequenas. Usou-se o intervalo de confiança (IC, 95\%) na estimação paramétrica dos atuais resultados comparado com os da literatura. Foi considerado resultado significante o que produzisse valor $\mathrm{p}<0,05$.

\section{RESULTADOS}

O número de pessoas estudadas foi 483 , sendo que um casal, após fazer o EEG, se negou a assinar o consentimento informado, tendo sido excluído da presente pesquisa. Do restante, $234(48,6 \%)$ eram do sexo masculino, o que não pode ser considerado diferente do feminino $(p=0,7)$. A mediana da idade foi de 21 (primeiro interquartil de 10 e terceiro de 37,5 ) conforme mostra a Figura 3.

A avaliação, que utilizou questionário padrão ouro $^{12}$ nos 24 casos positivos (Fig 2) apontados no rastreamento, identificou 16 casos falsos positivos. Destes, 9 foram de eventos não epilépticos psicogênicos (ENEP), 5 com crise única, 2 com convulsão febril e 1 com distúrbio de ritmo cardíaco. Dos 457 casos negativos rastreados, apenas um revelou-se

Tabela 1. Relação dos tipos de crises segundo a "International Classification of classification of epileptic seizures"11,15.

\begin{tabular}{|c|c|c|c|c|c|c|c|c|c|}
\hline \multicolumn{3}{|c|}{ Dados Pessoais } & \multicolumn{4}{|c|}{ Tipos de crises } & \multirow[b]{2}{*}{ Cron $^{17}$} & \multirow[b]{2}{*}{ Freq $^{6}$} & \multirow[b]{2}{*}{$\mathrm{DAE}^{7}$} \\
\hline & Idade & Sexo & $\mathrm{GEN}^{3}$ & Parcial & $\mathrm{Pr}^{4}$ & $\mathrm{NC}^{5}$ & & & \\
\hline 1 & 8 & $\mathrm{M}^{1}$ & - & - & & Sim & $1^{8}$ & - & sem \\
\hline 2 & 22 & $M$ & - & Indef ${ }^{10}$ & $\mathrm{P}^{11} \rightarrow \mathrm{TC}^{12}$ & - & $A^{9}$ & mensal & sem \\
\hline 3 & 36 & $\mathrm{~F}^{2}$ & - & PC & $\mathrm{PC}^{13} \rightarrow \mathrm{TC}$ & - & $A$ & semanal & sem \\
\hline 4 & 14 & $\mathrm{M}$ & - & Visual & $\mathrm{P} \rightarrow \mathrm{TC}$ & - & $A$ & mensal & sem \\
\hline 5 & 16 & M & - & - & $\mathrm{CT}$ & Sim & 1 & - & sem \\
\hline 6 & 1 & $\mathrm{~F}$ & atônica & - & - & - & $A$ & diária & $\mathrm{CLN}^{14}$ \\
\hline 7 & 37 & $\mathrm{~F}$ & - & $\mathrm{E}^{15}$ & $\mathrm{P} \rightarrow \mathrm{PC} \rightarrow \mathrm{TC}$ & - & A & mensal & $\mathrm{PB}+\mathrm{PHT}^{\mathrm{b}}$ \\
\hline 8 & 41 & $\mathrm{~F}$ & - & $\mathrm{S}^{16}$ & $\mathrm{P} \rightarrow \mathrm{TC}$ & - & 1 & - & $\mathrm{PB}^{\mathrm{a}}$ \\
\hline 9 & 2 & $\mathrm{~F}$ & - & - & - & Sim & $A$ & mensal & sem \\
\hline
\end{tabular}

${ }^{1}$ Masculino; ${ }^{2}$ Feminino; ${ }^{3}$ Generalizada; ${ }^{4}$ Propagação da crise; ${ }^{5}$ Crise não classificada; ${ }^{6}$ Frequência; ${ }^{7}$ Droga antiepiléptica; ${ }^{8}$ nativa; ${ }^{9}$ Ativa, ${ }^{10}$ Indefinida; ${ }^{11} \mathrm{Crise}$ parcial simples; ${ }^{12} \mathrm{Crise}$ tônico Clônica; ${ }^{13} \mathrm{Crise}$ parcial complexa; ${ }^{14} \mathrm{Clonazepan;}{ }^{\mathrm{a} F e n o b a r b i t a l ;} ;{ }^{17} \mathrm{Cronicidade;}{ }^{\mathrm{b}} \mathrm{Fenitoína;}$ ${ }^{15}$ Experiencial; ${ }^{16}$ Sensitiva/motora.

Tabela 2. Relação entre os epilépticos com e sem antecedentes familiares de epilepsia.

\begin{tabular}{lccc}
\hline & Com crise & Sem crises & Total \\
\hline Com antecedentes & 8 & 252 & 260 \\
Sem antecedentes & 1 & 220 & 221 \\
Total & 9 & 472 & 481 \\
\hline
\end{tabular}


falso negativo. A validação do questionário aplicado nessa população de 481 índios Bakairi mostrou sensibilidade de $88,9 \%$ e especificidade de $97,8 \%$.

Entre os 9 casos de epilepsia 5 (55\%) apresentavam crises parciais ( 1 complexa, 1 experiêncial, 1 visual, 1 sensitiva e 1 indefinida) e 3 (33,3\%) não classificadas e $1(11,1 \%)$ generalizada atônica (Tabela 1).

Os EEGs foram normais em todos os índios epilépticos estudados, exceto o caso de uma criança, atualmente com 9 meses e que, aos 3 meses, iniciaram-se as crises do tipo espasmos em flexão, parada do desenvolvimento, epilepsia generalizada do tipo crises atônicas diárias. Seu EEG mostrou atividade elétrica cerebral alentecida, com predomínio de ondas theta/delta pontiagudas de voltagem até $200 \mu \mathrm{V}$ além de descargas de poliponta difusa.

Apenas três epilépticos faziam uso regular de drogas anti-epilépticas (DAE), sendo que dois casos tinham crises parciais com evolução para crise tônico-clônica mensais, com mais de 20 anos de duração, faziam uso de politerapia com fenobarbital (100 $\mathrm{mg} / \mathrm{dia}$ ) fenitoína ( $200 \mathrm{mg} / \mathrm{dia}$ ) e um caso da síndrome de West que usava clonazepan ( $2 \mathrm{mg} / \mathrm{dia}$ ).

Cinco epilépticos apresentavam antecedentes familiares de epilepsia, ausentes outros fatores de risco como traumatismo crânio-encefálico (perda da consciência por mais de 30 minutos), alcoolismo, cisticercose, vascular e tumores cerebrais (Tabela 2).

\section{DISCUSSÃO}

Este estudo mostra uma prevalência de epilepsia de $18,6 \%$ entre os índios Bakairi, em 12,4\%o tratase de epilepsia ativa. Estudos, embora com metodologias diferentes, mas também com bases populacionais urbanas no Brasil, mostram resultados similares ${ }^{3,4}$. Gracia et al. ${ }^{6}$, usando metodologia semelhante, estudaram a prevalência de epilepsia na população dos índios Guaymis no Panamá. Encontraram a surpreendente prevalência de $57 \%$ o, sendo este fato relacionado ao fator de risco "antecedentes familiares"; em estudo na população urbana da cidade do Panamá, encontraram prevalência de epilepsia ativa de $22 \% 0^{20}$.

A prevalência de epilepsia de 18,6\%o encontrada neste estudo aproxima-se mais das encontradas nos países em desenvolvimento ${ }^{10,21-26}$ que nos industrializados ${ }^{16,27-30}$.

Era de se esperar que a prevalência de epilepsia entre os Bakairi estivesse abaixo da encontrada nos países em desenvolvimento, portanto mais próxima à dos países desenvolvidos, mesmo levando-se em conta o viés metodológico inerente a amostragens pequenas $^{31}$. Essa referida expectativa deve-se aos seguintes fatos: a população estudada tem assistência de saúde pública satisfatória, serviços de assistência de natalidade e puericultura; população jovem (idade média de 25,5 anos, com o primeiro quartil em 10 e o terceiro em 37,5 anos), reduzida população de idosos (menos de 2,5\% pessoas acima de 70 anos); ausência de acidentes de trânsito e sem guerras tribais há mais de 40 anos como causa de traumatismos cranianos; pelo fato de não haver culturalmente o hábito de se criar porcos para alimentação entre esse povo, a neurocisticercose não constitui fator de risco para a epilepsia como acontece nos estados ao sul do Brasil, segundo Bittencourt et al. ${ }^{8}$. Porém, não se fez exames de imagem e/ou de líquor cefalorraqueano. $\mathrm{O}$ alcoolismo não constitui problema para a população em tela.

O possível fator de risco que explicaria a alta prevalência encontrada entre os índios Bakairi fica por conta do antecedente familial, conforme os números da Tabela $2(p=0,04)$. Tal fato decorre da grande consanguinidade existente entre os índios Bakairi. Três fatos reforçam essa hipótese: 1) a população, há 60 anos, girava em torno de 140 indivíduos $^{10,32}$ e, atualmente, é composta por aproximadamente 546 indivíduos; 2) não houve miscigenação significante com outros povos ${ }^{32}$, portanto essa significante expansão populacional se deve, sobretudo, aos casamentos entre membros dessa comunidade. Corroboram esta verificação, ainda, os dois casos registrados, entre os casos falsos positivos, de crise convulsiva febril que também tem ocorrência com tendência familiar.

Os índios Bakairi têm pleno conhecimento do que é uma crise convulsiva, mas não das crises epilépticas não convulsivas. As convulsões são tratadas, segundo os rituais de sua cultura. Acreditam que durante o período da concepção até o primeiro ano de vida, os pais não podem matar e nem se alimentar de determinados animais como o tatu, a paca, o veado, porque que as mioclonias pré-morte (convulsão) passariam para a criança por vingança dos espíritos tutores de cada espécie dos animais abati$\operatorname{dos}^{10,11}$. Mas, também são encaminhadas para tratamento médico convencional presente na própria aldeia. Estes aspectos, acrescidos do fato de eles falarem fluentemente o português, serem dóceis e colaborantes foram relevantes na realização e conclusão dos resultados deste trabalho.

A principal limitação deste estudo foi o pequeno número de indivíduos na população, o que ocasionou uma reduzida amostra de indivíduos com epilep- 
sia. Estudos em comunidade indígena, por outro lado, abrem a possibilidade de se abordar pessoas epilépticas virgens de tratamento medicamentoso, o que não acontece nas demais comunidades tidas como civilizadas, propiciando o conhecimento sobre evolução natural dessa doença conforme Sander tem alertado ${ }^{31}$. De qualquer forma, o estudo apresentou benefício imediato à população estudada, uma vez que dois índios sem diagnóstico de epilepsia foram diagnosticados e medicados, e o medicamento foi prontamente disponibilizado pelo Instituto Trópicus.

A presente pesquisa alerta sobre a alta prevalência de epilepsia nos índios Bakairi e este fato, provavelmente, seja devido ao fator de risco familial, uma vez que os outros fatores de riscos de epilepsia estão ausentes. Espera-se que estudos futuros com outras Nações indígenas brasileiras possibilitem a comparação dos atuais resultados para que estes achados sejam abordados de forma científica, minimizando o sofrimento causado pela epilepsia nessa minoria étnica brasileira, sem entretanto interferir e mesmo obstruir seus costumes quanto às abordagens xamânicas.

Agradecimentos - Agradecemos ao laboratório LUCOR Eletromedicina Ltda. que forneceu o eletrencefalógrafo digital e o engenheiro técnico para realização dos 50 exames na aldeia.

\section{REFERÊNCIAS}

1. Steidele S, Flugel D, Bauer J, Elger CE. Epilepsy in the very old. Fortschr Neurol Psycgiatr 1997;65:237-242.

2. Almeida N Filho. Epidemiologia social das epilepsias no Brasil. In Sena PG (ed). Novas achegas sobre a epilepsia. Salvador: UFBa, Centro Editorial e Didático, 1980:57-76.

3. Marino R Jr, Cukiert A, Pinho E. Aspectos epidemiológicos da epilepsia em São Paulo. Arq Neuropsiquiatr 1986;44:243-254.

4. Fernandes GJ, Sander JWAS. Epidemiologia e história natural das epilepsias. In Da Costa JC, Palmini A, Yacubian EMT, Cavalheiro EG. Fundamentos neurobiológicos das epilepsias: aspectos clínicos e cirúrgicos. São Paulo: Lemos, 1998:3-20.

5. Younis YO. Epidemiology of epilepsy among school populations in Khartoum Province, Sudan. J Trop Med Hyg 1983;86:213-216.

6. Gracia F, Lao SL, Castilho L, et al. Epidemiology of epilepsy in Guayami Indians from Bocas del Toro Province, Republic of Panama. Epilepsia 1990;31:718-723.

7. Gudmundson G. Epilepsy in Iceland. Acta Neurol Scand 1966;43 (Suppl.25):119-124

8. Bittencourt PRM, Adamolekum B, Bharucha N, et al. Epilepsy in tropics: II. Clinical presentation, pahthophysiology, immunologic, economics, and therapy. Epilepsia 1996;37:1128-1137.

9. Placencia M, Sander JWAS, Shorvon SD, Ellison RH, Cascante S. Validation of a screening questionnaire for the detection of epileptic seizures in epidemiological studies. Brain 1992;115:783-794.
10. Barros EP. Os Kurâ-Bakairi: saúde, doença e cosmologia. In Barros EP (ed.). Estudos de antropologia da doença entre os Bororo e os KurâBakairi. Cuiabá: EdUFMT, 1997:39-80.

11. Taukane D. Educação escolar entre os Kurâ-Bakairi. Dissertação de Mestrado, Instituto de Educação da Universidade Federal de Mato Grosso, Cuiabá, 1996.

12. Borges MA, De Marchi NSA, Sato AK, Aleixo FV, Cordeiro AJ. As síndromes e crises epilépticas (ILAE) suas relações com o trabalho: estudo prospectivo ambulatorial de 412 pacientes. Arq Neuropsiquiatr 2000;58:691-697.

13. Rose HT, Penry JK, Markush RE, Radlof LA, Putnum PL. Prevalence of epilepsy in children. Epilepsia 1973;14:133-152.

14. Commission on Classification and Terminology of the International League Against Epilepsy: proposal for revised clinical and electroencephalographic classification of epileptic seizures. Epilepsia 1981;22:489501.

15. Commission on Classification and Terminology of the International League against Epilepsy. Proposal for revised classification of epilepsies syndromes. Epilepsia 1989;30:389-399.

16. Jacoby A, Baker GA, Steen N, Potts P, Chadwick QW. The clinical course of epilepsy and its psychosocial correlates: findings from a U.K. community study. Epilepsia 1996;37:148-161.

17. Palmini A, Da Costa JC. Introdução à epileptologia clínica e classificação das epilepsias e crises epilépticas. In: Da Costa JC, Palmini A, Yacubian EMT, Cavalheiro ES (eds). Fundamentos neurobiológicos das epilepsias. São Paulo: Lemos, 1998:149-161.

18. Commission on Epidemiology and Prognosis, International League Against Epilepsy: guidelines for epidemiologic studies on epilepsy. Epilepsia 1993;34:592-596.

19. Semah F, Picor C, Adam C, et al. Is the underlying cause of epilepsy a major prognostic factor for recurrence? Neurology 1998;51:1256-1262.

20. Gracia FJ, Bayard V, Trana E, et al. Prevalence of neurologic diseases in Belisario Porras municipality, district of San Miguelito, Panama, 1986. Med Panama 1988;13:40-45.

21. Osuntokun BO, Adeuja OG, Nottidge VA, et al. Prevalence of epilepsies in Nigerian Africans: a community-based study. Epilepsia 1987;28:272-279.

22. LiS, Schoenberg BS, Wang C, Cheng X, Zhou S, Bolis CL. Epidemiology of epilepsy in urban areas of the People's Republic of China. Epilepsia 1985;26:391-394.

23. Bharucha NE, Bharucha EP, Bharucha AE, Bhise AV, Schoenberg BS Prevalence of epilepsy in the Parsi community of Bombay. Epilepsia 1988;29:111-115.

24. Carpio A, Morales J, Calle H, Tinoco L, Santillan F. Prevalencia de epilepsia en la parroquia Cumbe, Azuay, Ecuador . Revista del Instituto de Ciencias de Salud Universidade de Cuenca, Ecuador 1986;1:10-31.

25. García-Pedroza F, Rubio-Donnadieu F, Garcia -Ramos G, EscobedoRíos F, Gonzáles-Cortéz A. Prevalence of epilepsy in children: Tlapan, Mexico City, Mexico. Neuroepidemiology 1983;2:16-23.

26. Placencia M, Suarez J, Crespo F, et al. A large scale study of epilepsy in Ecuador: methodological aspects. Neuroepidemiology 1992;11:74-84.

27. Zielinski JJ. Epidemiology and medico-social problems of epilepsy in Warsaw: final report on Research Program N¹9-P-58325-F01. Warsaw: Psychoneurological Institute, 1974:50-55.

28. Hauser WA, Annegers JF, Rocca WA. Descriptive epidemiology of epilepsy: contribution of population-based studies from Rochester, Minnesota. Mayo Clin Proc 1996;71:576-586.

29. Hauser WA. Incidence and prevalence. In Engel J, Pedley TA (eds.) Epilepsy: comprehensive textbook. Philadelphia: Lippincott-Raven, 1997:47-52.

30. Waaler PE, Blom BH, Skeidsvoll H, Mykletun. Prevalence, classification, and severity of epilepsy in children in western Norway. Epilepsia 2000;41:802-810.

31. Sander JWAS. Some aspects of prognosis in the epilepsies: a review. Epilepsia 1993;34:1007-1016.

32. Barros EP. História e Cosmologia na Organização de um Povo Karíb: os Bakairi. Tese de Doutorado, Universidade de São Paulo. São Paulo, 1992. 